\title{
IODETO DE POTÁSSIO SUPORTADO EM PENEIRAS MOLECULARES MESOPOROSAS (SBA-15 E MCM-41) COMO CATALISADOR BÁSICO PARA SÍNTESE DE BIODIESEL
}

\author{
Luzia Patricia Fernandes de Carvalho Galvão*, Marcela Nascimento Barbosa, Antonio Souza Araujo e Valter José \\ Fernandes Júnior \\ Instituto de Química, Universidade Federal do Rio Grande do Norte, 59072-970 Natal - RN, Brasil \\ Anne Gabriela Dias Santos \\ Departamento de Química, Universidade do Estado do Rio Grande do Norte, 59610-210 Mossoró - RN, Brasil \\ Geraldo E. Luz Jr. \\ Departamento de Química, Universidade Estadual do Piauí, Campus Poeta Torquato Neto, 64002-150 Teresina - PI, Brasil
}

Recebido em 31/12/10; aceito em 10/5/11; publicado na web em 8/7/11

\begin{abstract}
POTASSIUM IODINE SUPPORTED ON MESOPOROUS MOLECULAR SIEVES (SBA-15 AND MCM-41) AS BASIC CATALYST FOR SYNTHESIS OF BIODIESEL. Iodide potassium incorporated on mesoporous molecular sieves (SBA-15 and MCM-41) was used as heterogeneous catalysts in the transesterification of sunflower oil under different conditions of reaction time and ratio catalyst/oil (w/w). The results have showed that the system supported in SBA-15 has been more active than the supported in MCM-41, promoting a conversion to methyl esters of $84.98 \%$.
\end{abstract}

Keyword: mesoporous materials; transesterification; heterogeneous catalyst.

\section{INTRODUÇÃO}

Os óleos vegetais têm sido considerados fontes de biomassa, disponíveis para serem usados no lugar de óleo diesel. No entanto, embora favorável energeticamente, os óleos vegetais têm uma alta viscosidade e baixa volatilidade, características que dificultam o bom funcionamento dos motores. Além disso, a presença de resíduos de gomas e outros causam o bloqueio dos filtros, linhas e injetores. Associado a estes problemas, a combustão incompleta forma acroleína, muito prejudicial à saúde humana, e alguns óleos apresentam baixos índices de cetano, o que impede que essa fonte de energia seja utilizada diretamente como combustível. ${ }^{1,2}$

Dentro deste contexto, como uma ótima alternativa de biocombustível, tem-se o biodiesel, um combustível renovável e biodegradável, que consiste de uma mistura de ésteres metílicos ou etílicos de ácidos graxos, que normalmente é obtido pela transesterificação de triglicerídeos com álcool de cadeia curta e um catalisador. ${ }^{3}$ A reação de transesterificação pode ser realizada sob catálise homogênea ou heterogênea, podendo estas serem ácida ou básica. A catálise homogênea básica tem sido a mais comumente usada, devido à sua velocidade e eficiência. No entanto, esta rota catalítica apresenta alguns inconvenientes, como a difícil separação do catalisador ao final da reação e a produção indesejada de sabões, que diminuem o rendimento da reação e dificultam o processo de separação da glicerina do biodiesel e sua posterior purificação. ${ }^{4,5}$ Devido a estas características do processo homogêneo, têm sido intensificados os estudos sobre o processo catalítico heterogêneo. Algumas das vantagens deste último processo são a redução do número de etapas de purificação e a facilidade de separação do catalisador do biodiesel e da glicerina, podendo ser usado em reações posteriores. Alguns catalisadores sólidos, básico ou ácido, foram testados na reação de transesterificação de triglicerídeos com metanol. ${ }^{6,7}$ Os catalisadores básicos apresentaram taxas de reações maiores do que os sólidos ácidos e, desta forma, têm sido estudados com maior ênfase. ${ }^{8}$ Nesta classe de catalisadores, óxidos, hidróxidos e alcóxidos de metais alcalinos terrosos têm tido suas atividades

*e-mail: patriciagalvao.ufrn@gmail.com catalíticas avaliadas na obtenção de biodiesel por transesterificação de triglicerídeos. ${ }^{9}$ Dentre estes, os óxidos metálicos são os mais estudados pelo fato de possuírem na sua estrutura átomos metálicos com densidade de carga positiva, que se comportam como ácidos de Lewis, e átomos de oxigênio com densidade de carga negativa, que se comportam como uma de Brönsted. No entanto, a aplicação de tais compostos como catalisadores heterogêneos na síntese de biodiesel apresenta problemas devido à fácil carbonatação e lixiviação parcial dos mesmos no meio reacional.

Uma das maneiras de minimizar tal processo de lixiviação é ancorar o óxido em um suporte catalítico. Neste contexto, as peneiras moleculares mesoporosas do tipo MCM-41 e SBA-15 apresentam-se como promissores suportes catalíticos para o desenvolvimento de novos catalisadores heterogêneos, com fases ativas e bem dispersas. Estas peneiras moleculares apresentam elevada área superficial, grande diâmetro de poros e consideráveis estabilidades térmica e hidrotérmica, além de apresentarem poros unidirecionais. Alguns trabalhos relatam a obtenção de catalisadores mesoporosos pela incorporação de óxidos alcalinos e alcalino-terrosos, ou moléculas orgânicas básicas, na superfície dos materiais mesoporosos. ${ }^{10-13}$

Este trabalho teve por objetivo sintetizar, caracterizar e estudar a incorporação de iodeto de potássio em materiais mesoporosos do tipo MCM-41 e SBA-15, bem como avaliar as suas atividades catalíticas na reação de transesterificação de óleo de girassol em diferentes condições de reação.

\section{PARTE EXPERIMENTAL}

\section{Preparação dos catalisadores}

As peneiras moleculares mesoporosas, SBA-15 e MCM-41, foram sintetizadas respectivamente de acordo com Coutinho et al. ${ }^{14} \mathrm{e}$ Araujo et al. ${ }^{15}$ Estes suportes foram impregnados pelo método via úmido com soluções aquosas de iodeto de potássio. ${ }^{16}$ Para tanto, $50 \mathrm{~mL}$ de uma solução diluída de iodeto de potássio, a $35 \%$ em peso, foram misturados a 1,0 g de suporte. Em seguida, esse sistema teve sua água reduzida por meio de agitação, a $80^{\circ} \mathrm{C}$, sob pressão ambiente. Após 
secagem em estufa a $100{ }^{\circ} \mathrm{C}$, o sólido impregnado foi calcinado a 500 ${ }^{\circ} \mathrm{C}$ sob fluxo de ar $\left(100 \mathrm{~mL} \mathrm{~min}^{-1}\right)$ por $3 \mathrm{~h}$. Os catalisadores assim obtidos foram denominados KI/SBA-15 e KI/MCM-41.

\section{Caracterização dos catalisadores}

Os difratogramas de raios-X da SBA-15 e da MCM-41 foram obtidos em um equipamento da Shimadzu XRD 6000, utilizando radiações de $\mathrm{CuK} \alpha$ e filtro de níquel com uma voltagem e corrente do tubo de $30 \mathrm{kV}$ e $30 \mathrm{~mA}$, respectivamente. Estes difratogramas foram obtidos na faixa de $2 \theta$ de $0,5-5^{\circ}$ para SBA-15 e $1-10^{\circ}$ para MCM-41. Em relação aos catalisadores suportados (KI/SBA-15 e $\mathrm{KI} / \mathrm{MCM}-41$ ), os difratogramas foram obtidos de forma semelhante, tanto no intervalo $2 \theta$ de $0,5-5^{\circ}$, como no intervalo de $2 \theta$ de $10-90^{\circ}$, para avaliar a presença alguma fase cristalina incorporada.

As análises de adsorção e dessorção de nitrogênio foram obtidas a uma temperatura de $-196{ }^{\circ} \mathrm{C}$ em um equipamento ASAP-2010 da Micromeritics. Antes das medidas de adsorção cada amostra foi desgaseificada à temperatura de $300{ }^{\circ} \mathrm{C}$ por $2 \mathrm{~h}$. A área superficial específica foi determinada pelo método BET e volume de poros total pelo método de ponto único. A distribuição do tamanho de poros foi obtida pelo método Barrett-Joyner-Halena (BJH).

A basicidade dos catalisadores foi determinada através da análise volumétrica utilizando uma solução padrão de biftalato ácido de potássio $0,1 \mathrm{~mol} \mathrm{~L}^{-1}$ e fenolftaleína como indicador. ${ }^{17}$

\section{Transesterificação}

As reações de transesterificação foram realizadas utilizando óleo de girassol e metanol na razão molar de 15:1, usando como catalisadores o KI/MCM-41 e KI/SBA-15, ambas nas seguintes concentrações: 1 e $2 \%$ em peso. Todas as reações foram realizadas em um reator de vidro de $250 \mathrm{~mL}$, acoplado com um condensador de refluxo e um agitador magnético, sob pressão atmosférica. A temperatura de reação foi $60{ }^{\circ} \mathrm{C}$ e o tempo reacional de 4 h. Após a reação, o catalisador foi separado por filtração e o biodiesel foi lavado com água e seco a $100{ }^{\circ} \mathrm{C} .{ }^{18} \mathrm{Um}$ procedimento semelhante foi realizado para cada catalisador a fim de avaliar o efeito do tempo reacional no rendimento. $\mathrm{O}$ processo descrito foi repetido utilizando $1 \%$ em peso dos catalisadores, alterando o tempo de reação de 6 e 8 h.

\section{Caracterização do biodiesel}

A conversão dos triglicerídeos em ésteres metílicos foi avaliada por cromatografia gasosa, em um equipamento CG Trace Ultra da Thermo pelo método NBR 15343.

As curvas termogravimétricas (TG/DTG) foram obtidas em uma termobalança Mettler, modelo STGA 851. As análises térmicas foram realizadas sob fluxo de hélio $\left(25 \mathrm{~mL} \mathrm{~min}^{-1}\right)$ da temperatura ambiente até $600{ }^{\circ} \mathrm{C}$ com uma taxa de aquecimento de $10^{\circ} \mathrm{C} \mathrm{min}{ }^{-1}$.

\section{RESULTADOS E DISCUSSÃO}

\section{Caracterização dos catalisadores}

Os difratogramas de Raios-X das peneiras moleculares mesoporosas, SBA-15 e MCM-41, estão apresentados nas Figuras 1(A) e (B), respectivamente. Ambos difratogramas apresentam três picos de difração referentes aos planos de difração (100), (110) e (200), característicos da estrutura mesoporosa bidimensional com simetria P6mm, como aquela presente em materiais do tipo SBA15 e MCM-41.
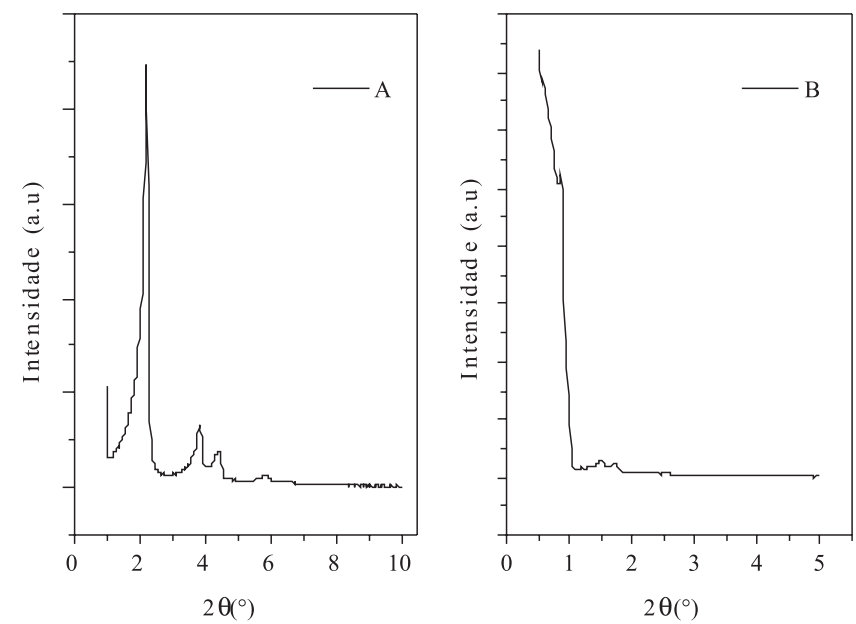

Figura 1. Difratograma de raios-X de MCM-41(A) e SBA-15 (B)

Nas Figuras 2 (A) e (B) estão apresentados os difratogramas de Raios-X dos sólidos catalíticos KI/SBA-15 e KI/MCM-41, respectivamente. Observa-se que somente o difratograma do sólido KI/SBA-15 apresentou os picos de difração das fases cristalinas referentes ao $\mathrm{KI}$ e $\mathrm{K}_{2} \mathrm{O}$, este último formado durante a calcinação da peneira molecular impregnada com KI. Não foi observada a presença destes picos de difração no sólido KI/MCM-41. Em relação ao KI, este fato pode indicar uma menor ou não formação de cristais com tamanho suficiente para promover a difração dos raios-X. Esta também pode ser uma explicação para a não observação de picos de difração relativos ao $\mathrm{K}_{2} \mathrm{O}$ no sólido $\mathrm{KI} / \mathrm{MCM}-41$, assim como a menor conversão do $\mathrm{KI}$ em $\mathrm{K}_{2} \mathrm{O}$, durante a calcinação, quando compara à amostra KI/SBA-15.

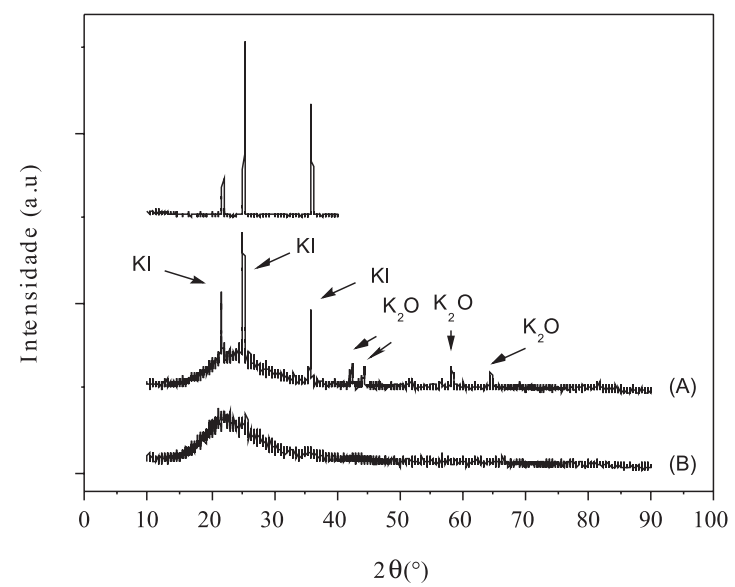

Figura 2. Difratograma com $2 \theta$ na faixa de 10 até $90^{\circ}$ para as amostras $A$ (KI/SBA-15) e para as amostras B (KI/MCM-41)

Os resultados de adsorção/dessorção de nitrogênio, apresentados na Tabela 1, demonstram que houve uma redução no volume de poros das peneiras moleculares mesoporosas, SBA-15 e MCM-41, quando $\mathrm{KI}$ e $\mathrm{K}_{2} \mathrm{O}$ foram incorporados em suas estruturas, sendo estas reduções mais intensas no suporte SBA-15. Observa-se também que o suporte SBA-15 teve seu diâmetro de poros reduzido em decorrência da incorporação do $\mathrm{KI} / \mathrm{K}_{2} \mathrm{O}$. Os dois fatos citados podem indicar que tais compostos tenham sido ancorados à superfície interna, dentro dos mesoporos, do referido suporte. Já para o suporte MCM-41, esta incorporação não promoveu alteração no diâmetro de poros, indicando uma incorporação na superfície externa do mesmo. 
Tabela 1. Propriedades texturais das silicas mesoporosas calcinadas a $773 \mathrm{~K}$

\begin{tabular}{lccc}
\hline Amostra & $\mathrm{Dp}(\mathrm{nm})$ & $\mathrm{Vp}\left(\mathrm{cm}^{3} \mathrm{~g}^{-1}\right)$ & $\mathrm{S}_{\mathrm{BET}}\left(\mathrm{m}^{2} \mathrm{~g}^{-1}\right)$ \\
\hline MCM-41 & 2,00 & 0,13 & 998 \\
SBA-15 & 4,33 & 1,08 & 932 \\
KI/MCM-41 & 2,00 & 0,04 & 14 \\
KI/SBA-15 & 1,60 & 0,05 & 25 \\
\hline
\end{tabular}

Outro fato observado na Tabela 1 foi a redução da área superficial dos suportes mesoporosos, decorrente da incorporação de $\mathrm{KI} / \mathrm{K}_{2} \mathrm{O}$. Além disso, observa-se que a referida redução foi mais intensa na MCM-41, cuja área superficial variou de 998 para 14 m²/g, após a incorporação. Tais fatos podem ser relacionados ao desordenamento da estrutura de poros dos suportes após a incorporação de $\mathrm{KI} / \mathrm{K}_{2} \mathrm{O}$, que, como mencionado, foi mais intenso na MCM-41.

A basicidade dos sólidos catalíticos, apresentada na Tabela 2, corrobora os resultados de DRX já apresentados e, a semelhança destes, indica uma maior conversão do $\mathrm{KI}$ em $\mathrm{K}_{2} \mathrm{O}$ na amostra $\mathrm{KI} /$ SBA-15, uma vez que a basicidade das amostras pode ser correlacionada à presença do $\mathrm{K}_{2} \mathrm{O}$.

Tabela 2. Basicidade e densidade dos sítios básicos dos catalisadores

\begin{tabular}{lcc}
\hline Amostras & $\begin{array}{c}\text { Basicidade } \\
(\mathrm{mmol} / \mathrm{g})\end{array}$ & $\begin{array}{c}\text { Densidade dos sítios } \\
\text { básicos }\left(\mathrm{mmol} / \mathrm{m}^{2}\right)\end{array}$ \\
\hline MCM-41 & - & - \\
SBA-15 & - & - \\
KI/MCM-41 & 0,31 & 0,03 \\
KI/SBA-15 & 0,75 & 0,03 \\
\hline
\end{tabular}

\section{Biodiesel}

As propriedades físico-químicas do óleo de girassol, utilizado como matéria-prima para reação de transesterificação, estão apresentadas na Tabela 3. Todas as propriedades estão de acordo com os parâmetros estabelecidos pela American Oil Chemists Society (AOCS).

Tabela 3. Propriedades físico-químicas do óleo de girassol

\begin{tabular}{lc}
\hline Propriedades físico-químicas & Óleo de girassol \\
\hline Viscosidade $\left(\mathrm{mm}^{2} / \mathrm{s}\right)$ & 33,2 \\
Tensão superficial $(\mathrm{mN} / \mathrm{m})$ & 18,1 \\
Massa específica a $20^{\circ} \mathrm{C}\left(\mathrm{kg} / \mathrm{m}^{3}\right)$ & 922,2 \\
Ponto de fulgor $\left(\mathrm{C}^{\circ}\right)$ & 314,6 \\
Ponto de combustão $\left({ }^{\circ} \mathrm{C}\right)$ & 320,0 \\
Índice de acidez $(\mathrm{mg} \mathrm{KOH} / \mathrm{g})$ & 0,032 \\
\hline
\end{tabular}

O teor de ésteres metílicos do biodiesel em diferentes condições de síntese é mostrado na Tabela 4. Para ambos os sólidos catalíticos utilizados, o aumento da concentração do catalisador de 1 a $2 \%$ promoveu um incremento na conversão dos triglicerídeos em ésteres metílicos. Em relação ao tempo de reação, os sólidos catalíticos mostraram diferentes comportamentos. O KI/MCM-41 promoveu uma conversão melhor com 6 h de reação. Neste sistema catalítico, a alteração do tempo de reação de 6 a 8 h promoveu uma diminuição na conversão, o que pode indicar que houve inversão da reação transesterificação, corroborada pela saturação dos sítios básicos do referido sistema catalítico. Já o sistema KI/SBA-15 promoveu uma maior conversão com $8 \mathrm{~h}$ de reação. Além disso, também foi observado que, em todas as condições, o KI/SBA-15 apresentou melhor atividade catalítica do que KI/MCM-41. Tais fatos podem ser explicados pela maior concentração de $\mathrm{K}_{2} \mathrm{O}$ no KI/SBA-15, em comparação com o KI/MCM-41, como indicada pelas análises de difração de raios-X. Além disso, a menor atividade catalítica do KI/MCM-41 pode estar relacionada também a sua menor área superficial e maior desordenamento da estrutura de poros, quando comparado ao KI/SBA-15.

Tabela 4. Composição dos ésteres metílicos

\begin{tabular}{lc}
\hline Catalisadores & Ésteres $(\%)$ \\
\hline $1 \% \mathrm{KI} / \mathrm{SBA}-154 \mathrm{~h}$ & 17,86 \\
$2 \% \mathrm{KI} / \mathrm{SBA}-154 \mathrm{~h}$ & 42,84 \\
$2 \% \mathrm{KI} / \mathrm{SBA}-156 \mathrm{~h}$ & 66,52 \\
$2 \% \mathrm{KI} / \mathrm{SBA}-158 \mathrm{~h}$ & 73,7 \\
$1 \% \mathrm{KI} / \mathrm{MCM}-414 \mathrm{~h}$ & 9,7 \\
$2 \% \mathrm{KI} / \mathrm{MCM}-414 \mathrm{~h}$ & 29,5 \\
$2 \% \mathrm{KI} / \mathrm{MCM}-416 \mathrm{~h}$ & 55,26 \\
$2 \% \mathrm{KI} / \mathrm{MCM}-418 \mathrm{~h}$ & 46,76 \\
\hline
\end{tabular}

As curvas TG das amostras de biodiesel obtidos com 2\% KI/ SBA-15 e 2\%KI/MCM-41 estão apresentados nas Figuras 3 e 4, respectivamente. Com o auxílio da Tabela 5 observa-se que em ambas as curvas há duas perdas de massa, relacionadas à volatilização dos ésteres metílicos e à decomposição dos triglicerídeos não convertidos, respectivamente. Além disso, para biodiesel obtido sobre 2\% KI/SBA15 , a primeira perda de massa aumentou com o aumento do tempo de reação, atingindo o valor máximo com a amostra obtida com $8 \mathrm{~h}$ de reação; enquanto que a segunda perda diminuiu. Já para biodiesel obtido sobre $2 \% \mathrm{KI} / \mathrm{MCM}-41$, a primeira perda de massa foi mais intensa na amostra obtida com $6 \mathrm{~h}$ de reação. Este comportamento pode ser associado à conversão dos triglicerídeos em ésteres metílicos, já que a primeira perda de massa é associada à volatilização destes últimos compostos, e, desta forma, estão em conformidade com os resultados obtidos por meio da análise cromatográfica das amostras de biodiesel.

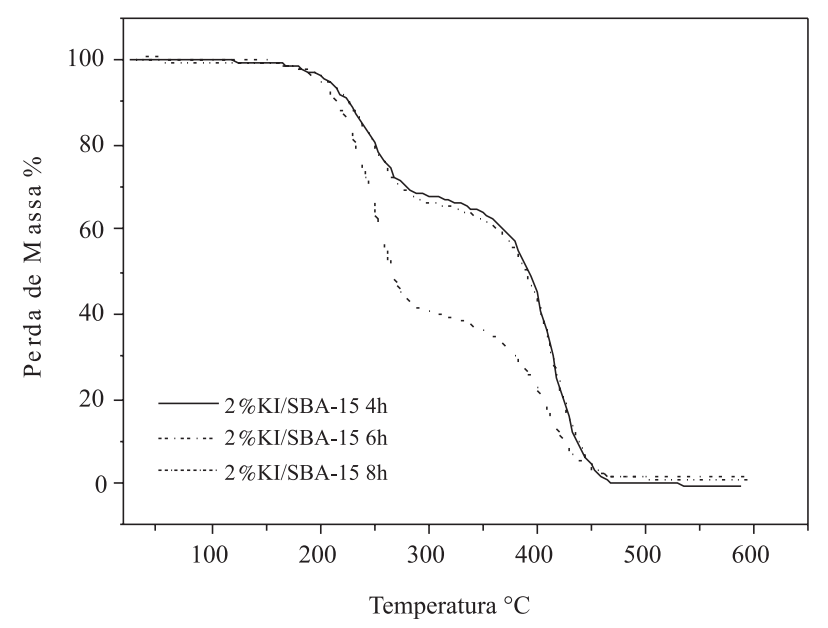

Figura 3. Curvas TG do biodiesel utilizando como catalisador $2 \%$ KI/SBA-15, nos tempos 4,6 e $8 \mathrm{~h}$

\section{CONCLUSÕES}

Em resumo, o catalisador KI/SBA-15 apresentou melhor atividade catalítica na reação de transesterificação de óleo de girassol do que 
Tabela 5. Intervalo de temperatura, temperatura do pico máximo e massa residual correspondente às curvas TG/DTG das amostras de biodiesel

\begin{tabular}{|c|c|c|c|c|c|c|}
\hline \multirow{2}{*}{ Amostras } & \multicolumn{2}{|c|}{$\mathrm{T}\left({ }^{\circ} \mathrm{C}\right)$} & \multicolumn{2}{|c|}{ Perda de massa $(\%)$} & \multicolumn{2}{|c|}{ Massa residual (\%) } \\
\hline & $1^{\circ}$ evento & $2^{\circ}$ evento & $1^{\circ}$ evento & $2^{\circ}$ evento & $1^{\circ}$ evento & $2^{\circ}$ evento \\
\hline Óleo de girassol & $265-480$ & & \multicolumn{2}{|c|}{96,8} & \multicolumn{2}{|c|}{3,2} \\
\hline KI/MCM-41 (4 h) & $135-305$ & $305-495$ & 8,4 & 90,4 & 91,6 & 1,2 \\
\hline KI/MCM-41 (6 h) & $140-305$ & $305-500$ & 40,3 & 59,7 & 57,5 & 2,2 \\
\hline KI/MCM-41 (8 h) & $100-320$ & $320-490$ & 28,5 & 71,5 & 72,7 & 1.2 \\
\hline KI/SBA-15 (4 h) & $140-310$ & $310-490$ & 31,9 & 67,4 & 68,1 & 0,7 \\
\hline KI/SBA-15 (6 h) & $105-320$ & $320-510$ & 34,7 & 64,5 & 65,2 & 0.7 \\
\hline KI/SBA-15 (8 h) & $100-310$ & $310-485$ & 60,1 & 38,4 & 39,9 & 1,5 \\
\hline
\end{tabular}

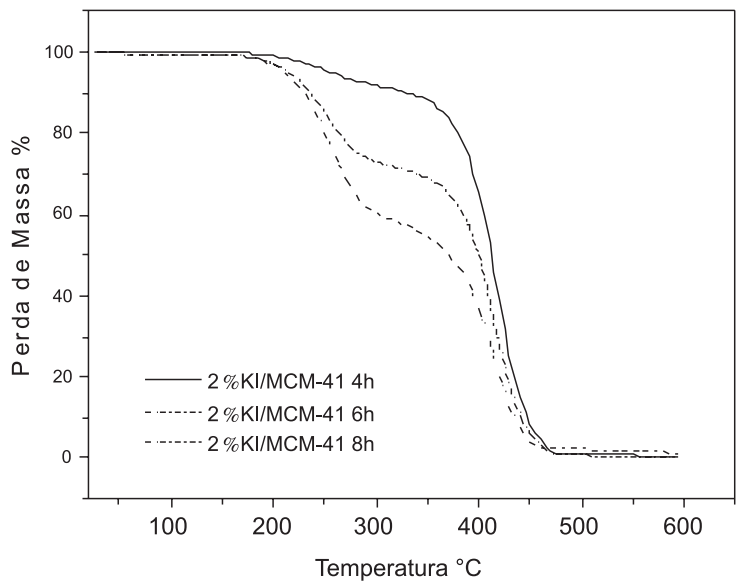

Figura 4. Curvas TG do biodiesel utilizando como catalisador $2 \%$ KI/MCM41, nos tempos 4,6 e $8 \mathrm{~h}$

o KI/MCM-41. Isto pode ser devido à formação de espécies ativas de $\mathrm{K}_{2} \mathrm{O}$, oriundas da decomposição térmica do $\mathrm{KI}$, observada nos difratogramas de raios- $\mathrm{X}$, bem como através da basicidade, cujo valor foi maior para KI/SBA-15 do que para KI/MCM-41. Portanto, a interação sal-suporte, nessas condições, resultou na geração de sítios básicos na superfície. Quanto à reação de transesterificação, $2 \%$ do catalisador KI/SBA-15 às $8 \mathrm{~h}$ apresentou melhor resultado na conversão de triglicerídeos em ésteres metílicos, que foi confirmada por cromatografia gasosa. No entanto, no que diz respeito à norma europeia EN 1403, que exige o conteúdo de ésteres de 96,5\%, as amostras não se encontram dentro das especificações. Outra observação importante foi a correlação qualitativa dos resultados da análise termogravimétrica com a cromatografia gasosa.

\section{AGRADECIMENTOS}

Ao apoio financeiro da ANP, PRH-30, CNPq, FINEP, UESPI e FAPEPI.

\section{REFERÊNCIAS}

1. Ryan III, W.; Dodge, L. G.; Callahan, T. J.; J. Am. Oil Chem. Soc. 1984, 61,1610

2. Schuchardt, U.; Lopes, O. C.; Anais do Simpósio Nacional sobre fontes renováveis de energia, Brasília, Brasil, 1988.

3. Liu, X.; He, H.; Wang, Y.; Zhu, S.; Catal. Commun. 2007, 8, 1107.

4. Agarwal, A. K.; Das, L. M. J.; Engin. Gás Turbines and Power 2001, $123,440$.

5. Encinar, J. M.; González, J. L.; Sábio, E.; Ramiro, M. J.; Ind. Eng. Chem. 1999, 38, 2927.

6. Garcia, C. M.; Teixeira, S.; Marciniuk, L. L.; Schuchardt, U.; Bioresour. Technol. 2008, 99, 6608.

7. Serio, M. Di; Ledda, M.; Cozzolino, M.; Minutillo, G.; Tesser, R.; Santacesaria, E.; Ind. Eng. Chem. Res. 2006, 45, 3009.

8. Venkat Reddy, C. R.; Oshel, R.; Verkade, J. G.; Energy Fuels 2006, 20, 1310

9. Leclercq, E.; Finiels, A.; Moreau, C. ; J. Am. Oil Chem. Soc. 2001, 78, 61.

10. Cantrell, D. G.; Gillie, L. J.; Lee, A. F.; Wilson, K.; Appl. Catal., A 2005 , 287, 183

11. Serio, M. Di; Cozzolino, M.; Giordano, M.; Tesser, R.; Patrono, P.; Santacesaria, E.; Ind. Eng. Chem. Res. 2006, 46, 6379.

12. Zhao, D.; Feng, J.; Huo, Q.; Melosh, N.; Fredrickson, G. H.; Chmelka, B. F.; Stucky, G. D.; Science 1998, 279.

13. Wang, Y. M.; Wu, Z. Y.; Wei, Y. L.; Zhu, J. H.; Microporous Mesoporous Mater. 2005, 84, 127.

14. Coutinho, A. C. S.; Souza, M. J. B.; Aquinio, J. M. F. B.; Silva, A. O. S.; Araujo, A. S.; Resumo dos Anais do Encontro Brasileiro sobre Adsorção, Natal, Brasil, 2004.

15. Araujo, A. S.; Jaroniec, M.; Thermochim. Acta 2000, 363, 175.

16. Xie, W.; Li, H.; J. Mol. Catal. A: Chem. 2006, 255, 1.

17. Araujo, A. S.; Junior, V. J. F.; Anais da Associação Brasileira de Química, Brasil, 1999.

18. Santos, A. G. D.; Araujo, A. S.; Caldeira, V. P. S.; Fernandes Jr., V. J.; Souza, L. D.; Barros, A. K.; Thermochim. Acta 2010, 506, 57. 